\title{
Massive dual gravity in $N$ spacetime dimensions
}

\author{
H. Alshal ${ }^{a, b}$ and T.L. Curtright ${ }^{a}$ \\ ${ }^{a}$ Department of Physics, University of Miami, \\ Coral Gables, Florida 33124, U.S.A. \\ ${ }^{b}$ Department of Physics, Faculty of Science, Cairo University, \\ Giza, 12613, Egypt \\ E-mail: halshal@sci.cu.edu.eg, curtright@miami.edu
}

ABSTRACT: We describe a field theory for "massive dual gravity" in $N$ spacetime dimensions. We obtain a Lagrangian that gives the lowest order coupling of the field to the $N$-dimensional curl of its own energy-momentum tensor. We then briefly discuss classical solutions. Finally, we show the theory is the exact dual of the Ogievetsky-Polubarinov model generalized to any $N$.

KEYwords: Classical Theories of Gravity, Duality in Gauge Field Theories, Field Theories in Higher Dimensions

ArXIV EPRINT: 1907.11537 
The suggestion that the gravitational field might be massive was made long ago and has been studied in great detail by various authors, albeit for a very small mass with an extremely slow exponential fall-off for the corresponding Yukawa potential (e.g. see [1]). The subject was surveyed almost exhaustively in [2-4]. However, that survey completely overlooked at least one interesting possibility.

Consider a field theory dual to that of a massive symmetric tensor $\left(h_{\mu \nu}=h_{\nu \mu}\right)$ in $N$ spacetime dimensions (i.e. "ND"), as an extension of the ideas and results in [5-8]. For ND the dual field of $h_{\mu \nu}$ is another tensor field whose rank depends on whether the original $h_{\mu \nu}$ tensor is massless or massive. If massless the dual of $h_{\mu \nu}$ is a tensor $T_{\left[\lambda_{1} \cdots \lambda_{N-3}\right] \mu}$ of rank $N-2$, while if massive the dual of $h_{\mu \nu}$ is a tensor $T_{\left[\lambda_{1} \cdots \lambda_{N-2}\right] \mu}$ of rank $N-1$. The indices for these $T$ fields are symmetrized, in an obvious way, according to the corresponding Young tableaux. ${ }^{1}$ Various individual fields of this type appear in string theories [9-12], and in "M-theory" and "E-theory" [13-17]. For a recent review of duality for gravity and higher-spin fields, with an emphasis on massless models in higher dimensions, see [18].

As a preliminary check, the number of on-shell degrees of freedom ("dof") for these different fields are as follows, when considered to be irreducible (hence traceless) tensor representations of $O(N-2)$ and $O(N-1)$ for the massless and massive cases, respectively, as computed using the well-known hook-length ${ }^{2}$ rules and the Schur-Weyl theorem. ${ }^{3}$

$$
\begin{aligned}
\operatorname{dof}\left(h_{\mu \nu}\right) & =\frac{(N-2)(N-1)}{2}-1=\frac{N(N-3)}{2} \quad \text { if massless. } \\
\operatorname{dof}\left(T_{\left[\lambda_{1} \cdots \lambda_{N-3}\right] \mu}\right) & =\frac{(2) \cdots(N-2)(N-1)}{(1) \cdots(N-4)(N-2)(1)}-\frac{(3) \cdots(N-2)}{(1) \cdots(N-4)}=\frac{N(N-3)}{2} \quad \text { if massless. } \\
\operatorname{dof}\left(h_{\mu \nu}\right) & =\frac{(N-1)(N)}{2}-1=\frac{(N+1)(N-2)}{2} \quad \text { if massive. } \\
\operatorname{dof}\left(T_{\left[\lambda_{1} \cdots \lambda_{N-2}\right] \mu}\right) & =\frac{(2) \cdots(N-1)(N)}{(1) \cdots(N-3)(N-1)(1)}-\frac{(3) \cdots(N-1)}{(1) \cdots(N-3)}=\frac{(N+1)(N-2)}{2} \quad \text { if massive. }
\end{aligned}
$$

Thus the degrees of freedom agree for the respective cases. For the massive situation in $N$ dimensions, the ranks and dofs of fields are well-known to coincide with those for the massless situation in $N+1$ dimensions. On the other hand, the dynamics of the fields require less trivial considerations.

For massive gravity the dual free field Lagrangian density is given by $[5-8,19]$

$$
\mathcal{L}=\mathcal{K}_{\mu}{ }_{\mu} \mathcal{K}_{\nu}^{\mu}+\frac{(-1)^{N} m^{2}}{(N-2) !}\left(T_{\left[\lambda_{1} \cdots \lambda_{N-2}\right] \mu} T^{\left[\lambda_{1} \cdots \lambda_{N-2}\right] \mu}-(N-2) T_{\left[\lambda_{1} \cdots \lambda_{N-3}\right]} T^{\left[\lambda_{1} \cdots \lambda_{N-3}\right]}\right)
$$

\footnotetext{
${ }^{1}$ https://en.wikipedia.org/wiki/Young_tableau.

${ }^{2}$ https://en.wikipedia.org/wiki/Hook_length_formula.

${ }^{3}$ https://en.wikipedia.org/wiki/Schur-Weyl_duality.
} 
with a choice of the overall normalization, and with the definitions

$$
\begin{aligned}
F_{\left[\lambda_{1} \cdots \lambda_{N-1}\right] \mu} & \equiv \partial_{\lambda_{1}} T_{\left[\lambda_{2} \cdots \lambda_{N-1}\right] \mu} \pm\left\{N-2 \text { signed permutations of } \lambda^{\prime} \mathrm{s}\right\} \\
K_{\mu}{ }^{\nu} & \equiv F_{\left[\lambda_{1} \cdots \lambda_{N-1}\right] \mu} \varepsilon^{\lambda_{1} \cdots \lambda_{N-1} \nu}=(N-1) \partial_{\lambda_{1}} T_{\left[\lambda_{2} \cdots \lambda_{N-1}\right] \mu} \varepsilon^{\lambda_{1} \cdots \lambda_{N-1} \nu} \\
\mathcal{K}_{\mu}{ }^{\nu} & \equiv \frac{1}{(N-1) !} K_{\mu}{ }^{\nu}, \quad T_{\left[\lambda_{1} \cdots \lambda_{N-3}\right]} \equiv T_{\left[\lambda_{1} \cdots \lambda_{N-3} \mu\right] \nu} \eta^{\mu \nu},
\end{aligned}
$$

where the Lorentz metric is $\eta_{\mu \nu}=\operatorname{diag}(+1,-1, \cdots,-1)$. Some $N$-dependent coefficients have been incorporated into the definition of $\mathcal{K}_{\mu}^{\nu}$ to take into account the number of antisymmetrized summed indices in the definition of $K_{\mu}{ }^{\nu}$.

It is instructive to compare $\mathcal{L}$ to the previously studied $4 \mathrm{D}$ case [5-8].

$$
\begin{aligned}
\mathcal{L}_{4 D} & =-\frac{1}{6}\left(F_{[\lambda \mu \nu] \rho} F^{[\lambda \mu \nu] \rho}-3 F_{[\mu \nu]} F^{[\mu \nu]}\right)+\frac{1}{2} m^{2}\left(T_{[\lambda \mu] \nu} T^{[\lambda \mu] \nu}-2 T_{\lambda} T^{\lambda}\right) \\
& =\mathcal{K}_{\mu}{ }^{\nu} \mathcal{K}_{\nu}{ }^{\mu}+\frac{1}{2} m^{2}\left(T_{[\lambda \mu] \nu} T^{[\lambda \mu] \nu}-2 T_{\lambda} T^{\lambda}\right) .
\end{aligned}
$$

This agrees with (3) for $N=4$. The reader should consider $N=3$ for a simpler example.

The free field equations are summarized in appendix A. A consistent interacting field equation for the massive ND model is an obvious generalization of the $4 \mathrm{D}$ equation [5-8], namely,

$$
\left(\square+m^{2}\right) T_{\left[\lambda_{1} \cdots \lambda_{N-2}\right] \nu}=\kappa P_{\lambda_{1} \cdots \lambda_{N-2} \nu, \alpha \beta \gamma} \partial^{\alpha} \Theta^{\beta \gamma},
$$

where a symmetrizer is defined to be

$$
\begin{aligned}
P_{\lambda_{1} \cdots \lambda_{N-2} \nu, \alpha \beta \gamma}= & (N-2) \varepsilon_{\lambda_{1} \cdots \lambda_{N-2} \alpha \beta} \eta_{\gamma \nu} \\
& +\varepsilon_{\nu \lambda_{2} \cdots \lambda_{N-2} \alpha \beta} \eta_{\gamma \lambda_{1}}+\varepsilon_{\lambda_{1} \nu \lambda_{3} \cdots \lambda_{N-2} \alpha \beta} \eta_{\gamma \lambda_{2}}+\cdots+\varepsilon_{\lambda_{1} \cdots \lambda_{N-3} \nu \alpha \beta} \eta_{\gamma \lambda_{N-2}},
\end{aligned}
$$

and where $\Theta_{\mu \nu}$ is any conserved, symmetric tensor, e.g. the energy-momentum tensor, and $\kappa$ is a dimensionful parameter with units $1 / m^{N / 2}$ since dimensionally $[T]=\frac{1}{2}(N-2)$ in mass units. It is natural to express $\kappa$ in terms of Newton's constant in ND, and a lengthscale set by the size of the envisioned ND universe, similar to the expression in 4D [5-8]. The r.h.s. of (8) is obtained below, to $O(\kappa)$, from a Lagrangian.

The field equation (8) implies that the trace $T_{\left[\lambda_{1} \cdots \lambda_{N-3}\right]}=\eta^{\nu \lambda_{N-2}} T_{\left[\lambda_{1} \cdots \lambda_{N-2}\right] \nu}$ and all divergences of $T_{\left[\lambda_{1} \cdots \lambda_{N-2}\right] \nu}$ decouple, i.e. they are free fields, and therefore they may be consistently set to zero leaving on-shell states that comprise only a single irreducible $S O(N-1)$ representation of mass $m$. For example, when $N=4$ unadulterated massive spin 2 states are obtained on-shell.

The on-shell field equation for the $T$-field strength $\mathcal{K}_{\mu}{ }^{\nu}$ is

$$
\left(\square+m^{2}\right) \mathcal{K}_{\mu}^{\nu}=\kappa\left((1-N) \square \Theta_{\mu}^{\nu}+\left(\delta_{\mu}^{\nu} \square-\partial_{\mu} \partial^{\nu}\right) \Theta\right) .
$$

This on-shell result for the $\mathcal{K}$-tensor follows from (8) and the identity

$$
\varepsilon^{\lambda \lambda_{1} \cdots \lambda_{N-2} \nu} P_{\lambda_{1} \cdots \lambda_{N-2} \mu, \alpha \beta \gamma}=(N-2) !\left((2-N) \delta_{\alpha \beta}^{\lambda \nu} \eta_{\gamma \mu}+\delta_{\mu \alpha \beta}^{\lambda \sigma \nu} \eta_{\gamma \sigma}\right) .
$$

In principle, there appear to be no fundamental barriers to prevent obtaining the field equations (8) and (10) from a closed-form Lagrangian for self-coupled dual fields, with the 
sources given to all orders in $\kappa$. Such is the case for the massive dual scalar field [7] (also see appendix $\mathrm{C}$ ). But it will suffice here to do this only to lowest order in $\kappa$.

The massive free field energy-momentum tensor,

$$
\begin{aligned}
\theta_{\mu}{ }^{\nu}= & \mathcal{K}_{\mu}{ }^{\lambda} \mathcal{K}_{\lambda}{ }^{\nu}+\frac{(-1)^{N-1} m^{2}}{(N-3) !} T_{\left[\mu \alpha_{2} \cdots \alpha_{N-2}\right] \lambda} T^{\left[\nu \alpha_{2} \cdots \alpha_{N-2}\right] \lambda} \\
& -\frac{1}{2} \delta_{\mu}{ }^{\nu}\left(\mathcal{K}_{\alpha \beta} \mathcal{K}^{\beta \alpha}-\frac{(-1)^{N} m^{2}}{(N-2) !} T_{\left[\alpha_{1} \cdots \alpha_{N-2}\right] \gamma} T^{\left[\alpha_{1} \cdots \alpha_{N-2}\right] \gamma}\right),
\end{aligned}
$$

is symmetric and conserved on-shell given the $O\left(\kappa^{0}\right)$ field equations, as discussed in the appendices. To obtain the field equations $(8)$ and (10) to $O(\kappa)$ this energy-momentum tensor must be augmented by adding a manifestly conserved $\left(\partial^{\mu} \vartheta_{\mu}^{\nu} \equiv 0\right)$, symmetric $\left(\vartheta_{\mu \nu}=\vartheta_{\nu \mu}\right)$ "improvement", namely,

$$
\begin{aligned}
\Theta_{\mu}{ }^{\nu}= & \theta_{\mu}{ }^{\nu}+\frac{(-1)^{N-1}}{(N-3) !} \vartheta_{\mu}^{\nu}, \\
\vartheta_{\mu}{ }^{\nu} \equiv & \square\left(T_{\left[\mu \alpha_{2} \cdots \alpha_{N-2}\right] c} T^{\left[\nu \alpha_{2} \cdots \alpha_{N-2}\right] c}\right)+\delta_{\mu}{ }^{\nu} \partial_{a} \partial^{b}\left(T^{\left[a \alpha_{2} \cdots \alpha_{N-2}\right] c} T_{\left[b \alpha_{2} \cdots \alpha_{N-2}\right] c}\right) \\
& -\partial_{\mu} \partial^{b}\left(T_{\left[b \alpha_{2} \cdots \alpha_{N-2}\right] c} T^{\left[\nu \alpha_{2} \cdots \alpha_{N-2}\right] c}\right)-\partial^{\nu} \partial_{b}\left(T^{\left[b \alpha_{2} \cdots \alpha_{N-2}\right] c} T_{\left[\mu \alpha_{2} \cdots \alpha_{N-2}\right] c}\right) .
\end{aligned}
$$

A Lagrangian which gives the sought-for field equation to $O(\kappa)$ (but unfortunately, not to $\left.O\left(\kappa^{2}\right)\right)$ is then obtained by adding to the massive free field Lagrangian $(3) O(\kappa)$ interactions suggested by the form $\mathcal{K}_{\alpha}{ }^{\beta} \Theta_{\beta}^{\alpha}$, namely,

$$
\begin{aligned}
\mathcal{L}_{\text {int }}= & \frac{1}{3}(-1)^{N-1}(N-1) ! \kappa \mathcal{K}_{\alpha}^{\beta} \mathcal{K}_{\beta}^{\gamma} \mathcal{K}_{\gamma}^{\alpha} \\
& +\frac{(-1)^{N-1} \kappa}{(N-3) !} T_{\left[\lambda_{1} \cdots \lambda_{N-2}\right] \nu} P^{\lambda_{1} \cdots \lambda_{N-2} \nu, \alpha \beta}{ }_{\gamma} \partial_{\alpha}\left(\begin{array}{c}
\left(\square+m^{2}\right)\left(T_{\left[\beta \alpha_{2} \cdots \alpha_{N-2}\right] c} T^{\left[\gamma \alpha_{2} \cdots \alpha_{N-2}\right] c}\right) \\
-\partial^{\gamma} \partial_{b}\left(T^{\left[b \alpha_{2} \cdots \alpha_{N-2}\right] c} T_{\left[\beta \alpha_{2} \cdots \alpha_{N-2}\right] c}\right)
\end{array}\right),
\end{aligned}
$$

up to a relative normalization between $\mathcal{L}$ and $\mathcal{L}_{\text {int }}$. The resulting action due to $\mathcal{L}_{\text {int }}$ is of course

$$
\mathcal{A}_{\text {int }}=\int \mathcal{L}_{\text {int }} d^{N} x
$$

and therefore, by varying $T^{\left[\lambda_{1} \cdots \lambda_{N-2}\right] \nu}$ in $\mathcal{A}_{\text {int }}$, the contributions to the field equations follow from

$$
\begin{aligned}
\delta \mathcal{A}_{\text {int }} & =\frac{(-1)^{N-1} \kappa}{((N-1) !)^{2}} \int\left(\delta K_{\alpha}^{\beta}\right) K_{\beta}^{\gamma} K_{\gamma}{ }^{\alpha} d^{N} x \\
& +\frac{(-1)^{N-1} \kappa}{(N-3) !} \int\left(\delta T_{\left[\lambda_{1} \cdots \lambda_{N-2}\right] \nu}\right) P^{\lambda_{1} \cdots \lambda_{N-2} \nu, \alpha \beta}{ }_{\gamma} \partial_{\alpha}\left(\begin{array}{c}
\left(\square+m^{2}\right)\left(T_{\left[\beta \alpha_{2} \cdots \alpha_{N-2}\right] c} T^{\left[\gamma \alpha_{2} \cdots \alpha_{N-2}\right] c}\right) \\
-\partial^{\gamma} \partial_{b}\left(T^{\left[b \alpha_{2} \cdots \alpha_{N-2}\right] c} T_{\left[\beta \alpha_{2} \cdots \alpha_{N-2}\right] c}\right)
\end{array}\right) d^{N} x \\
& +\frac{(-1)^{N-1} \kappa}{(N-3) !} \int T_{\left[\lambda_{1} \cdots \lambda_{N-2}\right] \nu} P^{\lambda_{1} \cdots \lambda_{N-2} \nu, \alpha \beta}{ }_{\gamma} \partial_{\alpha}\left(\begin{array}{c}
\left(\square+m^{2}\right) \delta\left(T_{\left[\beta \alpha_{2} \cdots \alpha_{N-2}\right] c} T^{\left[\gamma \alpha_{2} \cdots \alpha_{N-2}\right] c}\right) \\
-\partial^{\gamma} \partial_{b} \delta\left(T^{\left[b \alpha_{2} \cdots \alpha_{N-2}\right] c} T_{\left[\beta \alpha_{2} \cdots \alpha_{N-2}\right] c}\right)
\end{array}\right) d^{N} x
\end{aligned}
$$

Upon integrating by parts the terms in the last line give no contributions to the bulk field equations at $O(\kappa)$ because of the $O\left(\kappa^{0}\right)$ on-shell conditions (cf. (A.9) and (A.6) in appendix A). These terms are important at $O\left(\kappa^{2}\right)$, but they have no effect at $O(\kappa)$. 
After integrating by parts, the bulk variation of the $K$ trilinear becomes

$$
\begin{aligned}
& \frac{(-1)^{N-1} \kappa}{((N-1) !)^{2}} \int\left(\delta K_{\alpha}^{\beta}\right) K_{\beta}^{\gamma} K_{\gamma}{ }^{\alpha} d^{4} x \\
& \quad=\frac{(-1)^{N-2}(N-1) \kappa}{((N-1) !)^{2}} \int\left(\delta T_{\left[\alpha_{2} \cdots \alpha_{N-1}\right] \alpha}\right) \varepsilon^{\alpha_{1} \cdots \alpha_{N-1} \beta} \partial_{\alpha_{1}}\left(K_{\beta}^{\gamma} K_{\gamma}{ }^{\alpha}\right) d^{N} x \\
& =\frac{\kappa}{((N-1) !)^{2}} \int\left(\delta T_{\left[\lambda_{1} \cdots \lambda_{N-2}\right] \nu}\right) P_{\gamma}^{\lambda_{1} \cdots \lambda_{N-2} \nu, \alpha \beta} \partial_{\alpha}\left(K_{\beta}{ }^{\mu} K_{\mu}^{\gamma}\right) d^{N} x
\end{aligned}
$$

where we have also exploited the symmetry of $T_{\left[\alpha_{2} \cdots \alpha_{N-1}\right] \alpha}$ and that of the symmetrizer to write

$$
(N-1)\left(\delta T_{\left[\alpha_{2} \cdots \alpha_{N-1}\right] \alpha}\right) \varepsilon^{\alpha_{1} \cdots \alpha_{N-1} \beta} \eta^{\alpha \gamma} \partial_{\alpha_{1}}=(-1)^{N-2}\left(\delta T_{\left[\lambda_{1} \cdots \lambda_{N-2}\right] \nu}\right) P^{\lambda_{1} \cdots \lambda_{N-2} \nu, \alpha \beta \gamma} \partial_{\alpha}
$$

The $O(\kappa)$ variation of the interaction is therefore

$$
\begin{aligned}
\delta \mathcal{A}_{\text {int }} & =\kappa \int\left(\delta T_{\left[\lambda_{1} \cdots \lambda_{N-2}\right] \nu}\right) P^{\lambda_{1} \cdots \lambda_{N-2} \nu, \alpha \beta} \partial_{\alpha}\left(\mathcal{K}_{\beta}^{\mu} \mathcal{K}_{\mu}^{\gamma}\right) d^{N} x \\
& +\frac{(-1)^{N-1} \kappa}{(N-3) !} \int\left(\delta T_{\left[\lambda_{1} \cdots \lambda_{N-2}\right] \nu}\right) P^{\lambda_{1} \cdots \lambda_{N-2} \nu, \alpha \beta}{ }_{\gamma} \partial_{\alpha}\left(\begin{array}{c}
\left(\square+m^{2}\right)\left(T_{\left[\beta \alpha_{2} \cdots \alpha_{N-2}\right] c} T^{\left[\gamma \alpha_{2} \cdots \alpha_{N-2}\right] c}\right) \\
-\partial^{\gamma} \partial_{b}\left(T^{\left[b \alpha_{2} \cdots \alpha_{N-2}\right] c} T_{\left[\beta \alpha_{2} \cdots \alpha_{N-2}\right] c}\right)
\end{array}\right) d^{N} x \\
& +\frac{(-1)^{N-1} \kappa}{(N-3) !} \int T_{\left[\lambda_{1} \cdots \lambda_{N-2}\right] \nu} P^{\lambda_{1} \cdots \lambda_{N-2} \nu, \alpha \beta} \partial_{\alpha}\left(\begin{array}{c}
\left(\square+m^{2}\right) \delta\left(T_{\left[\beta \alpha_{2} \cdots \alpha_{N-2}\right] c} T^{\left[\gamma \alpha_{2} \cdots \alpha_{N-2}\right] c}\right) \\
-\partial^{\gamma} \partial_{b} \delta\left(T^{\left[b \alpha_{2} \cdots \alpha_{N-2}\right] c} T_{\left[\beta \alpha_{2} \cdots \alpha_{N-2}\right] c}\right)
\end{array}\right) d^{N} x
\end{aligned}
$$

That is to say,

$$
\delta \mathcal{A}_{\text {int }}=\kappa \int\left(\delta T^{\left[\lambda_{1} \cdots \lambda_{N-2}\right] \nu}\right) P_{\lambda_{1} \cdots \lambda_{N-2} \nu, \alpha \beta \gamma} \partial^{\alpha} \Theta^{\beta \gamma} d^{N} x+O\left(\kappa^{2}\right)
$$

This variation thereby gives precisely the r.h.s. of the field equation (8) to lowest non-trivial order in $\kappa$.

Given that the r.h.s. of (8) is a total divergence, it may be somewhat surprising that energy-momentum can produce dual fields that are indistinguishable from conventional massive gravity solutions "outside the source" especially in the weak-field limit where the energy-momentum is due to sources other than the $T$-field itself. This is perhaps more easily seen from (10). In fact, that field equation is closely related to other, more familiar expressions.

Were it not for the manifestly conserved trace term, $\left(\partial_{\mu} \partial_{\nu}-\eta_{\mu \nu} \square\right) \Theta$, an obvious but naive inference from (10) would be that a more conventional form of massive gravity, such as that in [1], would be related to the on-shell dual theory just by the local identification ${ }^{4}$ $K_{\mu \nu} \propto \square h_{\mu \nu}$, where

$$
\left(\square+m^{2}\right) h_{\mu \nu}=\kappa \Theta_{\mu \nu}
$$

\footnotetext{
${ }^{4}$ A local identification $K_{\mu \nu}(x) \propto \square h_{\mu \nu}(x)$ would require a less palatable nonlocal inverse relation, $h_{\mu \nu}(x) \propto h_{\mu \nu}^{(0)}(x)+\int G(x, y) K_{\mu \nu}(y) d^{N} y$ where $G$ is a Green function such that $\square G(x, y)=\delta^{N}(x-y)$ and $h_{\mu \nu}^{(0)}$ is a free massless field.
} 
The trace term invalidates this simple identification, in general. Nevertheless, there are situations where the dual and conventional theories give equivalent results. This is especially true for static configurations.

Static sources do indeed produce $\mathcal{K}_{00}$ fields. In the weak-field limit where $T$-field dependence in $\Theta_{\mu \nu}$ can be ignored, the static equation is

$$
\left(\nabla^{2}-m^{2}\right) \mathcal{K}_{00}=-\kappa \nabla^{2}\left((N-1) \Theta_{00}-\Theta\right),
$$

an inhomogeneous equation with well-known solutions, for given static sources on the r.h.s. . That is to say,

$$
\left(\nabla^{2}-m^{2}\right) \mathcal{K}=-\mathcal{F}
$$

where $\mathcal{K}$ and $\mathcal{F}$ are defined by

$$
\mathcal{K}=\left(\mathcal{K}_{00}+\mathcal{F}\right) / m^{2}, \quad \mathcal{F}=\kappa(N-1) \Theta_{00}-\kappa \Theta .
$$

In regions where $\mathcal{F}=0$ (i.e. outside the source) then $\mathcal{K} \propto \mathcal{K}_{00}$.

Therefore, modulo boundary conditions, the solution for $\mathcal{K}$ would be the same as that for more conventional massive gravity, for an equivalent conventional source, namely, for $\kappa \Theta_{00}=\mathcal{F}$. Thus, outside the source in regions where $\mathcal{F}=0, h_{00}$ and $\mathcal{K}_{00}$ could easily be indistinguishable in the weak-field limit.

If $\Theta=0$ then clearly this indistinguishability could carry over to more general situations, including those with time dependence, since for vanishing energy-momentum trace,

$$
\left(\square+m^{2}\right) \mathcal{H}_{\mu \nu}=\kappa \Theta_{\mu \nu}
$$

with the field redefinition

$$
\mathcal{H}_{\mu \nu}=\frac{1}{m^{2}(N-1)}\left(\mathcal{K}_{\mu \nu}+\kappa(N-1) \Theta_{\mu \nu}\right) .
$$

So if $\Theta=0$ the field equation for $\mathcal{H}_{\mu \nu}$ coincides with that for $h_{\mu \nu}$. In this case, with suitable boundary conditions, the solutions would again be the same.

The preceding remarks suggest that (27) may be useful for the dual theory even when $\Theta \neq 0$ and even when the energy-momentum tensor includes contributions from the $T$-field itself so that the weak-field limit does not apply. In that case (27) is a nonlinear field redefinition that leads to the following equivalent restatement of (10).

$$
\left(\square+m^{2}\right) \mathcal{H}_{\mu \nu}=\kappa \Theta_{\mu \nu}+\frac{\kappa}{(N-1) m^{2}}\left(\eta_{\mu \nu} \square-\partial_{\mu} \partial_{\nu}\right) \Theta .
$$

From the first kinematic constraint in (A.5), the trace $\mathcal{H}=\mathcal{H}_{\mu}{ }^{\mu}$ is then fixed by (27) to be

$$
\mathcal{H}=\frac{\kappa}{m^{2}} \Theta
$$

This constraint on the trace is consistent with (28) because, given that field equation, the difference $\mathcal{H}-\kappa \Theta / m^{2}$ is a free field. Similarly, for conserved and symmetric $\Theta_{\mu \nu}$ both the divergence and antisymmetric parts of the $\mathcal{H}$-field are free and consistently set to zero. 
As stated above, (27) is in general a nonlinear field redefinition, given that $\Theta_{\mu \nu}$ will in general depend on the dual field, but in the weak-field limit, outside any non- $T$-field source of energy-momentum, the $\mathcal{H}_{\mu \nu}$ field is just proportional to $\mathcal{K}_{\mu \nu}$, hence proportional to the $T$-field strength. This is an expected relation that characterizes massive free (or weak) field duality: field and field strength are interchanged [6].

More importantly, as previously noted for the 4D case [8], the field equation (28) is not the conventional one in (22). That is to say, (8) and (22) are not massive duals of one another, in general. Rather, (28) is the ND extension of the field equation proposed by Ogievetsky and Polubarinov for a purely spin 2 massive field in 4D [20]. In that model $\mathcal{H}_{\mu \nu}$ would play the role of an elementary field, whereas in the theory described here $\mathcal{H}_{\mu \nu}$ is essentially the field strength of the dual $T$-field, albeit with some nonlinear embellishments due to the interaction. That is to say, the ND interacting massive $T$-theory described here is the exact dual of the ND Ogievetsky-Polubarinov model, with on-shell equivalence specified by (27).

More complete discussion of the phenomenological differences between the dual model given here and other massive gravity fields, for realistic source terms and sufficiently small values of $m^{2}$, will be given elsewhere.

\section{Acknowledgments}

We thank T.S. Van Kortryk for discussions, and especially for his concise contribution to appendix C. This work was supported in part by a University of Miami Cooper Fellowship.

\section{A Dual free field equations}

The bulk variation of the dual free field action is

$$
\begin{aligned}
\int \delta \mathcal{L} d^{N} x & =\int \frac{1}{((N-1) !)^{2}} \delta\left(K_{\mu \nu} K^{\nu \mu}\right) d^{N} x \\
& +\int \frac{(-1)^{N} m^{2}}{(N-2) !} \delta\left(T_{\left[\alpha_{1} \cdots \alpha_{N-2}\right] \mu} T^{\left[\alpha_{1} \cdots \alpha_{N-2}\right] \mu}-(N-2) T_{\left[\alpha_{1} \cdots \alpha_{N-3}\right]} T^{\left[\alpha_{1} \cdots \alpha_{N-3}\right]}\right) d^{N} x
\end{aligned}
$$

where we define ${ }^{5}$

$$
K_{\mu}^{\lambda}=F_{\left[\lambda_{1} \cdots \lambda_{N-1}\right] \mu} \varepsilon^{\lambda_{1} \cdots \lambda_{N-1} \lambda}=(N-1) \partial_{\alpha_{1}} T_{\left[\alpha_{2} \cdots \alpha_{N-1}\right] \mu} \varepsilon^{\alpha_{1} \cdots \alpha_{N-1} \lambda} .
$$

That is to say,

$$
\begin{aligned}
\int \delta \mathcal{L} d^{N} x= & \frac{2(-1)^{N-1}}{((N-1) !)^{2}} \int\left(\delta T_{\left[\alpha_{1} \cdots \alpha_{N-2}\right] \sigma}\right) P^{\left[\alpha_{1} \cdots \alpha_{N-2}\right] \sigma, \lambda \mu \nu} \\
& \times\left(\partial_{\lambda} K_{\mu \nu}+(N-1)(-1)^{N-1} m^{2} \varepsilon_{\nu \lambda \omega_{1} \cdots \omega_{N-2}} T^{\left[\omega_{1} \cdots \omega_{N-2}\right]}{ }_{\mu}\right) d^{N} x
\end{aligned}
$$

\footnotetext{
${ }^{5} \mathrm{NB} K_{\mu}{ }^{\lambda}=(N-1) ! \mathcal{K}_{\mu}{ }^{\lambda}$. Our rationale for using $K$ as well as $\mathcal{K}$ is to be consistent with the notation in $[8]$.
} 
Hence the dual free field equations, in raw form, are

$$
P^{\left[\alpha_{1} \cdots \alpha_{N-2}\right] \sigma, \lambda \mu \nu}\left(\partial_{\lambda} K_{\mu \nu}+(N-1)(-1)^{N-1} m^{2} \varepsilon_{\nu \lambda \omega_{1} \cdots \omega_{N-2}} T^{\left[\omega_{1} \cdots \omega_{N-2}\right]} \mu\right)=0,
$$

supplemented by the kinematic conditions,

$$
K_{\mu}^{\mu}=0, \quad \partial_{\nu} K_{\mu}^{\nu}=0
$$

By taking various divergences and contractions of (A.4), the field equations boil down to the following simplified "on-shell" conditions: the Klein-Gordon equation,

$$
\left(\square+m^{2}\right) T_{\left[\lambda_{1} \cdots \lambda_{N-2}\right] \mu}=0
$$

and the "half-shell" conditions,

$$
\begin{aligned}
T_{\left[\lambda_{1} \cdots \lambda_{N-3} \mu\right] \nu} \eta^{\mu \nu} & =0, \\
\partial_{\mu} T^{\left[\mu \lambda_{2} \cdots \lambda_{N-2}\right] \nu} & =0, \\
\partial_{\mu} T^{\left[\lambda_{1} \cdots \lambda_{N-2}\right] \mu} & =0 .
\end{aligned}
$$

Some immediate consequences of the half-shell conditions are: ${ }^{6}$

$$
\partial^{\nu} F_{\left[\lambda_{1} \cdots \lambda_{N-2} \mu\right] \nu} \bumpeq 0, \quad F_{\left[\lambda_{1} \cdots \lambda_{N-2} \mu\right] \nu} \eta^{\mu \nu} \bumpeq 0, \quad K_{\mu \nu} \bumpeq K_{\nu \mu}, \quad \partial^{\mu} K_{\mu \nu} \bumpeq 0 .
$$

For example, the third relation in (A.10) follows from the second since

$$
K^{\mu \nu}-K^{\nu \mu}=(-1)^{N-1}\left(F_{\left[\lambda_{1} \cdots \lambda_{N-1}\right]}^{\mu} \varepsilon^{\nu \lambda_{1} \cdots \lambda_{N-1}}-F_{\left[\lambda_{1} \cdots \lambda_{N-1}\right]}^{\nu} \varepsilon^{\mu \lambda_{1} \cdots \lambda_{N-1}}\right),
$$

and then by ND syzygy, ${ }^{7}$

$$
(-1)^{N-1}\left(F_{\left[\lambda_{1} \cdots \lambda_{N-1}\right]}^{\mu} \varepsilon^{\nu \lambda_{1} \cdots \lambda_{N-1}}-F_{\left[\lambda_{1} \cdots \lambda_{N-1}\right]}^{\nu} \varepsilon^{\mu \lambda_{1} \cdots \lambda_{N-1}}\right)=(N-1) F_{\left[\lambda_{1} \cdots \lambda_{N-2} \lambda\right]}^{\lambda} \varepsilon^{\mu \nu \lambda_{1} \cdots \lambda_{N-2}} .
$$

\section{B Free field energy-momentum conservation}

Conservation of $\theta_{\mu}{ }^{\nu}$ for the free theory is established by the following Lemmata.

\section{Lemma 1.}

$$
\partial^{\mu}\left(K_{\mu}{ }^{\lambda} K_{\lambda}{ }^{\nu}-\frac{1}{2} \delta_{\mu}{ }^{\nu} K_{\alpha \beta} K^{\beta \alpha}\right) \bumpeq(-1)^{N-2}(N-1) K_{\mu}{ }^{\lambda} \varepsilon^{\alpha_{1} \cdots \alpha_{N-2} \mu \nu} \square T_{\left[\alpha_{1} \cdots \alpha_{N-2}\right] \lambda} .
$$

\footnotetext{
${ }^{6}$ About the notation: as used in $[7,8]$, "乞 " means equality given one or more of the half-shell conditions, while " $\approx$ " means "full-shell" equality given the Klein-Gordon equation in addition to the half-shell conditions.

${ }^{7}$ http://mathworld.wolfram.com/Syzygy.html.
} 
Proof.

$$
\begin{aligned}
\partial^{\mu}\left(K_{\mu}{ }^{\lambda} K_{\lambda}{ }^{\nu}\right)= & K_{\lambda}{ }^{\nu} \partial^{\mu} K_{\mu}{ }^{\lambda}+K_{\mu}{ }^{\lambda} \partial^{\mu} K_{\lambda}{ }^{\nu} \bumpeq K_{\mu}{ }^{\lambda} \partial^{\mu} K_{\lambda}{ }^{\nu}=K_{\mu}{ }^{\lambda} \varepsilon^{\alpha_{1} \cdots \alpha_{N-1} \nu} \partial^{\mu} F_{\left[\alpha_{1} \cdots \alpha_{N-1}\right] \lambda} \\
& \operatorname{using}(\mathrm{A} .10) \text { and }(\mathrm{A} .2) \\
= & K_{\mu}{ }^{\lambda}\left(\varepsilon^{\alpha_{1} \cdots \alpha_{N-1} \mu} \partial^{\nu}+(N-1) \varepsilon^{\alpha_{1} \cdots \alpha_{N-2} \mu \nu} \partial^{\alpha_{N-1}}\right) F_{\left[\alpha_{1} \cdots \alpha_{N-1}\right] \lambda} \\
& \operatorname{syzygy}^{7} \text { in ND }[21,22] \\
\bumpeq & K_{\mu}{ }^{\lambda} \partial^{\nu} K_{\lambda}{ }^{\mu}+(-1)^{N-2}(N-1) K_{\mu}{ }^{\lambda} \varepsilon^{\alpha_{1} \cdots \alpha_{N-2} \mu \nu} \square T_{\left[\alpha_{1} \cdots \alpha_{N-2}\right] \lambda}
\end{aligned}
$$

where in the last step we have used (A.2) and (A.8), (A.9). So (B.1) is established. Thus we are led to

\section{Lemma 2.}

$$
K_{\mu}{ }^{\lambda} \varepsilon^{\alpha_{1} \cdots \alpha_{N-2} \mu \nu} \bumpeq(-1)^{N}(N-1) ! F^{\left[\alpha_{1} \cdots \alpha_{N-2} \nu\right] \lambda} .
$$

Proof.

$$
\begin{aligned}
K_{\mu}{ }^{\lambda} \varepsilon^{\alpha_{1} \cdots \alpha_{N-2} \mu \nu} & \bumpeq K_{\mu}^{\lambda} \varepsilon^{\alpha_{1} \cdots \alpha_{N-2} \mu \nu}=F^{\left[\lambda_{1} \cdots \lambda_{N-1}\right] \lambda} \varepsilon_{\lambda_{1} \cdots \lambda_{N-1} \mu} \varepsilon^{\alpha_{1} \cdots \alpha_{N-2} \mu \nu} \\
& =(-1)^{N} \delta_{\lambda_{1} \cdots \lambda_{N-1}}^{\alpha_{1} \cdots \alpha_{N-2} \nu} F^{\left[\lambda_{1} \cdots \lambda_{N-1}\right] \lambda}=(-1)^{N}(N-1) ! F^{\left[\alpha_{1} \cdots \alpha_{N-2} \nu\right] \lambda}
\end{aligned}
$$

So (B.2) is also established. Now, combining (B.1) and (B.2) along with (A.6) gives immediately

\section{Lemma 3.}

$$
\partial^{\mu}\left(K_{\mu}{ }^{\lambda} K_{\lambda}{ }^{\nu}-\frac{1}{2} \delta_{\mu}^{\nu} K_{\alpha \beta} K^{\beta \alpha}\right) \approx-(N-1)(N-1) ! m^{2} F^{\left[\alpha_{1} \cdots \alpha_{N-2} \nu\right] \lambda} T_{\left[\alpha_{1} \cdots \alpha_{N-2}\right] \lambda} .
$$

This leads to a final

\section{Lemma 4.}

$$
\begin{aligned}
& F^{\left[\alpha_{1} \cdots \alpha_{N-2} \nu\right] \lambda} T_{\left[\alpha_{1} \cdots \alpha_{N-2}\right] \lambda} \\
& =(-1)^{N}\left(\partial^{\nu}\left(\frac{1}{2} T_{\left[\alpha_{1} \cdots \alpha_{N-2}\right] \gamma} T^{\left[\alpha_{1} \cdots \alpha_{N-2}\right] \gamma}\right)-(N-2) \partial^{\mu}\left(T_{\left[\mu \alpha_{2} \cdots \alpha_{N-2}\right] \lambda} T^{\left[\nu \alpha_{2} \cdots \alpha_{N-2}\right] \lambda}\right)\right) .
\end{aligned}
$$

Proof.

$$
\begin{aligned}
& F^{\left[\alpha_{1} \cdots \alpha_{N-2} \nu\right] \lambda} T_{\left[\alpha_{1} \cdots \alpha_{N-2}\right] \lambda} \\
& =\left((-1)^{N-2} \partial^{\nu} T^{\left[\alpha_{1} \cdots \alpha_{N-2}\right] \lambda}+(N-2) \partial^{\alpha_{1}} T^{\left[\alpha_{2} \cdots \alpha_{N-2} \nu\right] \lambda}\right) T_{\left[\alpha_{1} \cdots \alpha_{N-2}\right] \lambda}
\end{aligned}
$$

definition of $F$

$$
\bumpeq \partial^{\nu}\left(\frac{1}{2}(-1)^{N} T_{\left[\alpha_{1} \cdots \alpha_{N-2}\right] \gamma} T^{\left[\alpha_{1} \cdots \alpha_{N-2}\right] \gamma}\right)+(N-2)(-1)^{N-3} \partial^{\mu}\left(T_{\left[\mu \alpha_{2} \cdots \alpha_{N-2}\right] \lambda} T^{\left[\nu \alpha_{2} \cdots \alpha_{N-2}\right] \lambda}\right)
$$

using (A.8)

So (B.4) is established. 
Combining (B.3) and (B.4) we then obtain

$$
\begin{aligned}
\begin{array}{l}
\partial^{\mu}\left(K_{\mu}{ }^{\lambda} K_{\lambda}{ }^{\nu}-\frac{1}{2} \delta_{\mu}{ }^{\nu} K_{\alpha \beta} K^{\beta \alpha}\right) \\
\approx-(-1)^{N}(N-1)(N-1) ! m^{2}(
\end{array} & \partial^{\nu}\left(\frac{1}{2} T_{\left[\alpha_{1} \cdots \alpha_{N-2}\right] \gamma} T^{\left[\alpha_{1} \cdots \alpha_{N-2}\right] \gamma}\right) \\
& \left.-(N-2) \partial^{\mu}\left(T_{\left[\mu \alpha_{2} \cdots \alpha_{N-2}\right] \lambda} T^{\left[\nu \alpha_{2} \cdots \alpha_{N-2}\right] \lambda}\right)\right) .
\end{aligned}
$$

That is to say, $\partial^{\mu} \theta_{\mu}^{\nu} \approx 0$ with $\theta_{\mu}^{\nu}$ given by

$$
\begin{aligned}
((N-1) !)^{2} \theta_{\mu}{ }^{\nu}= & K_{\mu}{ }^{\lambda} K_{\lambda}{ }^{\nu}-(N-1)(N-2)(N-1) !(-1)^{N} m^{2}\left(T_{\left[\mu \alpha_{2} \cdots \alpha_{N-2}\right] \lambda} T^{\left[\nu \alpha_{2} \cdots \alpha_{N-2}\right] \lambda}\right) \\
& -\frac{1}{2} \delta_{\mu}{ }^{\nu}\left(K_{\alpha \beta} K^{\beta \alpha}-(N-1)(N-1) !(-1)^{N} m^{2} T_{\left[\alpha_{1} \cdots \alpha_{N-2}\right] \gamma} T^{\left[\alpha_{1} \cdots \alpha_{N-2}\right] \gamma}\right) .
\end{aligned}
$$

The $N$-dependent factors make a little more sense when $\theta_{\mu}{ }^{\nu}$ is expressed in terms of $\mathcal{K}_{\mu}{ }^{\nu}$ :

$$
\begin{aligned}
\theta_{\mu}{ }^{\nu}= & \mathcal{K}_{\mu}{ }_{\mu} \mathcal{K}_{\lambda}{ }_{\lambda}-\frac{(-1)^{N} m^{2}}{(N-3) !} T_{\left[\mu \alpha_{2} \cdots \alpha_{N-2}\right] \lambda} T^{\left[\nu \alpha_{2} \cdots \alpha_{N-2}\right] \lambda} \\
& -\frac{1}{2} \delta_{\mu}{ }^{\nu}\left(\mathcal{K}_{\alpha \beta} \mathcal{K}^{\beta \alpha}-\frac{(-1)^{N} m^{2}}{(N-2) !} T_{\left[\alpha_{1} \cdots \alpha_{N-2}\right] \gamma} T^{\left[\alpha_{1} \cdots \alpha_{N-2}\right] \gamma}\right)
\end{aligned}
$$

But in any case, up to an overall numerical factor, all this agrees with the $4 \mathrm{D}$ results [5-8] when $N=4$ :

$$
\left.\theta_{\mu}^{\nu}\right|_{N=4} \propto K_{\mu \alpha} K^{\alpha \nu}-36 m^{2} T_{[\mu \beta] \gamma} T^{[\nu \beta] \gamma}-\delta_{\mu}{ }^{\nu}\left(\frac{1}{2} K_{\alpha \beta} K^{\beta \alpha}-9 m^{2} T_{[\alpha \beta] \gamma} T^{[\alpha \beta] \gamma}\right) .
$$

\section{Coupling a dual scalar field to $\Theta$}

In this appendix some $4 \mathrm{D}$ results for scalar fields $[7,8]$ are generalized to ND.

Consider a Lagrangian density $\mathcal{L}$ depending on a vector field $V^{\mu}$ through the two scalar variables,

$$
B=V_{\mu} V^{\mu}, \quad F=\partial_{\mu} V^{\mu} .
$$

The bulk field equations that follow from the action of $\mathcal{L}$ by varying $V^{\mu}$ are simply

$$
\partial_{\mu} \mathcal{L}_{F}=2 V_{\mu} \mathcal{L}_{B}
$$

where the partial derivatives of $\mathcal{L}$ are designated by $\mathcal{L}_{B} \equiv \partial \mathcal{L}(B, F) / \partial B$ and $\mathcal{L}_{F} \equiv$ $\partial \mathcal{L}(B, F) / \partial F$.

The vector field $V_{\mu}$ is to be understood as the $N$-dimensional spacetime dual of a totally antisymmetric, rank $N-1$, tensor gauge field, $V_{\alpha_{1} \cdots \alpha_{N-1}}$, with its corresponding totally antisymmetric, gauge invariant field strength, $F_{\mu \alpha_{1} \cdots \alpha_{N-1}}=\partial_{\mu} V_{\alpha_{1} \cdots \alpha_{N-1}} \pm N-1$ terms. Thus

$$
V^{\mu}=\frac{1}{(N-1) !} \varepsilon^{\mu \alpha_{1} \cdots \alpha_{N-1}} V_{\alpha_{1} \cdots \alpha_{N-1}}, \quad \partial_{\mu} V^{\mu}=\frac{1}{N !} \varepsilon^{\alpha_{1} \cdots \alpha_{N}} F_{\alpha_{1} \cdots \alpha_{N}} .
$$


Under massive field duality $[5,6]$, this field strength should become the gradient of a scalar $\Phi$,

$$
V_{\mu}=\partial_{\mu} \Phi
$$

such that

$$
\partial_{\mu} V_{\lambda}=\partial_{\lambda} V_{\mu} .
$$

The goal here is to find an $\mathcal{L}$ such that field equations for $V_{\mu}$ amount to (C.5) along with the "simple, indeed elegant" statement $[5,6]$,

$$
\left(\square+m^{2}\right) V_{\mu}=\kappa \partial_{\mu} \Theta,
$$

where $\Theta$ is the trace of the energy-momentum tensor for the $V$-field.

For simplicity, suppose $\mathcal{L}_{B}=a+b \mathcal{L}_{F}$ for constants $a$ and $b$, in accordance with $V_{\mu}$ being a gradient, as in (C.4) and (C.5). This linear condition is immediately integrated to obtain

$$
\mathcal{L}(B, F)=a B+L(F+b B),
$$

where $L(F+b B)$ is a differentiable function of the linear combination $F+b B$. The field equations (C.2) are now

$$
\partial_{\mu} L^{\prime}=2\left(a+b L^{\prime}\right) V_{\mu} .
$$

As is well-known, there may be two distinct expressions for energy-momentum tensors that result from any Lagrangian. From (C.7) the canonical results for $\Theta_{\mu \nu}$, and its trace $\Theta=\Theta_{\mu}^{\mu}$, are immediately seen to be

$$
\begin{aligned}
& \Theta_{\mu \nu}^{[\text {canonical }]}=\left(\partial_{\mu} V_{\nu}\right) L^{\prime}-g_{\mu \nu}(a B+L), \\
& \Theta^{[\text {canonical }]}=F L^{\prime}-N(a B+L) .
\end{aligned}
$$

Although not manifestly symmetric, it is nonetheless true that $\Theta_{\mu \nu}^{[\text {canonical] }}=\Theta_{\nu \mu}^{\text {[canonical] }}$ on-shell in light of the condition (C.5).

Surprisingly different results follow from covariantizing (C.7) with respect to an arbitrary background metric $g_{\mu \nu}$, varying the action for $\sqrt{\left|\operatorname{det} g_{\alpha \beta}\right|} \mathcal{L}$ with respect to that metric, and then taking the flat-space limit. This procedure gives the "gravitational" energy-momentum tensor and its trace:

$$
\begin{aligned}
& \Theta_{\mu \nu}^{[\text {gravitational }]}=-2\left(a+b L^{\prime}\right) V_{\mu} V_{\nu}-g_{\mu \nu}\left(L-a B-(F+2 b B) L^{\prime}\right), \\
& \Theta^{\text {[gravitational }]}=(N F+(2 N-2) b B) L^{\prime}+(N-2) a B-N L .
\end{aligned}
$$

The unusual structure exhibited in this tensor follows because, as defined by (C.3), $V^{\mu}$ is a relative contravariant vector ${ }^{8}$ of weight +1 with no dependence on the metric, so $\partial_{\mu} V^{\mu}$ is a relative scalar of weight +1 , also with no dependence on $g_{\mu \nu}$, and $V_{\mu} V^{\mu}=g_{\mu \nu} V^{\mu} V^{\nu}$ is a relative scalar of weight +2 where all dependence on the metric is shown explicitly. Hence the absolute scalar version of $\mathcal{L}(B, F)$ is given by

$$
\mathcal{L}=\frac{a g_{\mu \nu} V^{\mu} V^{\nu}}{\left|\operatorname{det} g_{\alpha \beta}\right|}+L\left(\frac{\partial_{\mu} V^{\mu}}{\sqrt{\left|\operatorname{det} g_{\alpha \beta}\right|}}+\frac{b g_{\mu \nu} V^{\mu} V^{\nu}}{\left|\operatorname{det} g_{\alpha \beta}\right|}\right),
$$

where once again all the metric dependence is shown explicitly.

\footnotetext{
${ }^{8}$ https://en.wikipedia.org/wiki/Tensor_density.
} 
It is straightforward to check on-shell conservation of either (C.9) or (C.10), separately. However, it turns out the flat-space equations of motion can now be written in the form (C.6) provided a linear combination of $\Theta_{\mu \nu}^{\text {[canonical] }}$ and $\Theta_{\mu \nu}^{\text {[gravitational] }}$ is used for the system's energy-momentum tensor. Let

$$
\Theta_{\mu \nu}=\frac{N-2}{N-1} \Theta_{\mu \nu}^{[\text {canonical }]}+\frac{1}{N-1} \Theta_{\mu \nu}^{\text {[gravitational }]} .
$$

The trace is then

$$
\Theta=\Theta_{\mu}^{\mu}=2(F+b B) L^{\prime}-N L-(N-2) a B .
$$

The field equations (C.5) and (C.8) give for the left-hand side of (C.6)

$$
\left(\square+m^{2}\right) V_{\mu}=\left(1+\frac{m^{2}}{2} \frac{L^{\prime \prime}}{a+b L^{\prime}}\right) \partial_{\mu}(F+b B)-b \partial_{\mu} B
$$

where (C.5) implies $\square V_{\mu}=\partial^{\lambda} \partial_{\lambda} V_{\mu}=\partial^{\lambda} \partial_{\mu} V_{\lambda}=\partial_{\mu} F$. On the other hand, from (C.13) for any constant $c$,

$$
c \partial_{\mu} \Theta=c\left(2(F+b B) L^{\prime \prime}-(N-2) L^{\prime}\right) \partial_{\mu}(F+b B)-(N-2) a c \partial_{\mu} B .
$$

The choice $(N-2) a c=b$ reconciles the spurious $\partial_{\mu} B$ term to give the desired form,

$$
\left(\square+m^{2}\right) V_{\mu}=c \partial_{\mu} \Theta
$$

provided the function $L$ satisfies the second-order nonlinear equation

$$
1+\frac{m^{2}}{2} \frac{L^{\prime \prime}(z)}{a+b L^{\prime}(z)}=c\left(2 z L^{\prime \prime}(z)-(N-2) L^{\prime}(z)\right) .
$$

Moreover, the constant $c$ can be set to a convenient nonzero value by a few scale changes.

For example, if $(a, L) \rightarrow\left(\frac{a b}{2 c}, \frac{a m^{2}}{2 b c} L\right)$, along with the previous choice $(N-2) a c=$ $b \rightarrow a=2 /(N-2)$, the equation for $L$ becomes

$$
1+\frac{m^{4}}{2 b} \frac{L^{\prime \prime}}{b+m^{2} L^{\prime}}=\frac{m^{2}}{b}\left(\frac{2}{(N-2)} z L^{\prime \prime}(z)-L^{\prime}(z)\right) .
$$

The rescaling $z \rightarrow m^{2} w / b$ then gives

$$
1+\frac{1}{2} \frac{L^{\prime \prime}}{1+L^{\prime}}=\frac{2}{(N-2)} w L^{\prime \prime}-L^{\prime},
$$

where the 's in (C.19) are $\frac{d}{d w}$ s. The solutions of this final differential equation are very dependent upon $N$. With the initial condition $L^{\prime}(0)=0$ a first integral is given by

$$
L^{\prime}=X-1,
$$

where $X$ is a root of

$$
X^{\frac{N}{N-2}}=\left(1-\frac{2 N}{(N-2)} w X\right)
$$

such that $X \rightarrow 1$ as $w \rightarrow 0$. 
The simplest cases of (C.21) are for $N=4$ and $N \rightarrow \infty$. In those cases, $(X)^{\frac{N}{N-2}}-\left.\left(1-\frac{2 N}{(N-2)} w X\right)\right|_{N=4}=X^{2}-1+4 w X$, so for $N=4$ the roots are: $\left\{X=\frac{1}{2}\left(-4 w+2 \sqrt{4 w^{2}+1}\right)\right\}$ and $\left\{X=\frac{1}{2}\left(-4 w-2 \sqrt{4 w^{2}+1}\right)\right\}$; while $N \rightarrow \infty$ gives just $X=(1-2 w X)$, whose solution is: $\left\{X=\frac{1}{1+2 w}\right\}$. Thus

$$
\begin{aligned}
\left.L^{\prime}(w)\right|_{N=4} & =\frac{1}{2}\left(2 \sqrt{1+4 w^{2}}-2-4 w\right), \\
\left.L^{\prime}(w)\right|_{N \rightarrow \infty} & =\frac{-2 w}{1+2 w} .
\end{aligned}
$$

For these two special cases a final integration with the initial condition $L(0)=0$ gives

$$
\begin{aligned}
\left.L(w)\right|_{N=4} & =-w-w^{2}+\frac{1}{2} w \sqrt{1+4 w^{2}}+\frac{1}{4} \ln \left(2 w+\sqrt{1+4 w^{2}}\right), \\
\left.L(w)\right|_{N \rightarrow \infty} & =-w+\frac{1}{2} \ln (1+2 w) .
\end{aligned}
$$

The first of these reproduces the result in $[7,8]$.

For other $N$ it might seem that things can get out of hand, except perhaps for $N=3$, 6, and 8. For the first two of these cases, (C.21) results in a cubic equation, which is tractable. For $N=8,(\mathrm{C} .21)$ is a quartic equation, which is also tractable. But for other $N$, (C.21) is quintic, or worse.

The general solution for these other values of $N$ is indeed nontrivial, but the Taylor series for $L^{\prime}$ is remarkably simple. For example,

$$
\begin{aligned}
L^{\prime}(w)= & -2 w+4 \frac{(N-3) w^{2}}{(N-2)}-\frac{8}{3}(N-4)(3 N-8) \frac{w^{3}}{(N-2)^{2}} \\
& +\frac{8}{3}(N-5)(2 N-5)(3 N-10) \frac{w^{4}}{(N-2)^{3}} \\
& -\frac{16}{15}(N-6)(2 N-6)(3 N-12)(5 N-12) \frac{w^{5}}{(N-2)^{4}}+O\left(w^{6}\right) .
\end{aligned}
$$

As a polynomial in $N$, the coefficient of $w^{m+1} /(N-2)^{m}$ always factors over the rationals. The complete series is [23]

$$
L^{\prime}(w)=\frac{N-2}{N} \sum_{m=1}^{\infty} \frac{1}{m !} \frac{\Gamma\left(\frac{N-2}{N}(1+m)\right)}{\Gamma\left(2-\frac{2}{N}(1+m)\right)}\left(\frac{2 N w}{2-N}\right)^{m} .
$$

This result for $L^{\prime}$ is a special case of Fox's generalized confluent hypergeometric function [24]. A final integration then yields the sought-for $\mathcal{L}$ for any $N$.

The field strength for the dual scalar obeys an equation that can be manipulated in a manner similar to that used in the main text to relate the dual gravitational field to the ND Ogievetsky-Polubarinov model. Taking the divergence of (C.6) gives

$$
\left(\square+m^{2}\right) F=\kappa \square \Theta
$$


A nonlinear field redefinition, namely,

$$
\Psi=\frac{1}{m^{2}}(F-\kappa \Theta),
$$

then converts (C.28) into

$$
\left(\square+m^{2}\right) \Psi=-\kappa \Theta .
$$

This is the field equation for the ND extended Freund-Nambu model [25] of a fundamental scalar field $\Psi$ coupled to the trace of its own energy-momentum tensor. That is to say, the $V_{\alpha_{1} \cdots \alpha_{N-1}}$ model constructed here is the massive dual of the Freund-Nambu scalar theory on-shell. In the latter model, of course, the trace is expressed as a local functional of $\Psi$, whereas $\Theta$ in (C.30) is a functional of $V_{\alpha_{1} \cdots \alpha_{N-1}}$ that must be re-expressed in terms of $\Psi$. That this can be done is perhaps not obvious, but nonetheless it is true.

Given the structural similarities between the Freund-Nambu theory and scalar gravitation [26], it is perhaps more plausible that the complete Lagrangian for the self-coupled $T_{\left[\lambda_{1} \cdots \lambda_{N-2}\right] \mu}$ field can be determined to all orders in $\kappa$.

Open Access. This article is distributed under the terms of the Creative Commons Attribution License (CC-BY 4.0), which permits any use, distribution and reproduction in any medium, provided the original author(s) and source are credited.

\section{References}

[1] P.G.O. Freund, A. Maheshwari and E. Schonberg, Finite Range Gravitation, Astrophys. J. $157(1969) 857$.

[2] J.B. Pitts and W.C. Schieve, Universally coupled massive gravity. I., Theor. Math. Phys. 151 (2007) 700 [gr-qc/0503051] [INSPIRE].

[3] J.B. Pitts, Universally Coupled Massive Gravity, II: Densitized Tetrad and Cotetrad Theories, Gen. Rel. Grav. 44 (2012) 401 [arXiv:1110.2077] [INSPIRE].

[4] J.B. Pitts, Universally coupled massive gravity, III: dRGT-Maheshwari pure spin-2, Ogievetsky-Polubarinov and arbitrary mass terms, Annals Phys. 365 (2016) 73 [arXiv: 1505.03492] [INSPIRE].

[5] T. Curtright, Generalized gauge fields, Phys. Lett. 165B (1985) 304 [InSPIRE].

[6] T.L. Curtright and P.G.O. Freund, Massive dual fields, Nucl. Phys. B 172 (1980) 413 [INSPIRE].

[7] T.L. Curtright, Massive Dual Spinless Fields Revisited, arXiv:1907.11530 [INSPIRE].

[8] T.L. Curtright and H. Alshal, Massive Dual Spin 2 Revisited, arXiv:1907.11532 [INSPIRE].

[9] T.L. Curtright and C.B. Thorn, Symmetry Patterns in the Mass Spectra of Dual String Models, Nucl. Phys. B 274 (1986) 520 [InSPIRE].

[10] T.L. Curtright, C.B. Thorn and J. Goldstone, Spin Content of the Bosonic String, Phys. Lett. B 175 (1986) 47 [INSPIRE].

[11] T.L. Curtright, G.I. Ghandour and C.B. Thorn, Spin Content of String Models, Phys. Lett. B 182 (1986) 45 [INSPIRE]. 
[12] T.L. Curtright, Counting symmetry patterns in the spectra of strings, in String Theory, Quantum Cosmology and Quantum Gravity, Integrable and Conformal Invariant Theories, Proceedings of the Paris-Meudon Colloquium, 22-26 September (1986), World Scientific (1987) [ITP-SB-86-74] [INSPIRE].

[13] C.M. Hull, Strongly coupled gravity and duality, Nucl. Phys. B 583 (2000) 237 [hep-th/0004195] [INSPIRE].

[14] C.M. Hull, Duality in gravity and higher spin gauge fields, JHEP 09 (2001) 027 [hep-th/0107149] [INSPIRE].

[15] P.C. West, $E_{11}$ and $M$-theory, Class. Quant. Grav. 18 (2001) 4443 [hep-th/0104081] [INSPIRE].

[16] P. West, A brief review of E theory, Int. J. Mod. Phys. A 31 (2016) 1630043 [arXiv: 1609.06863] [INSPIRE].

[17] T. Damour, M. Henneaux and H. Nicolai, $E_{10}$ and a 'small tension expansion' of M-theory, Phys. Rev. Lett. 89 (2002) 221601 [hep-th/0207267] [INSPIRE].

[18] A. Danehkar, Electric-Magnetic Duality in Gravity and Higher-Spin Fields, Front. Phys. 6 (2019) 146 [INSPIRE].

[19] B. Gonzalez, A. Khoudeir, R. Montemayor and L.F. Urrutia, Duality for massive spin two theories in arbitrary dimensions, JHEP 09 (2008) 058 [arXiv:0806.3200] [INSPIRE].

[20] V.I. Ogievetsky and I.V. Polubarinov, Interacting field of spin 2 and the Einstein equations, Annals Phys. 35 (1965) 167.

[21] D. Hilbert, Über die Theorie der algebraischen Formen, Math. Ann. 36 (1890) 473.

[22] J.J. Sylvester, On a Theory of Syzygetic Relations..., Phil. Trans. Roy. Soc. Lond. 143 (1853) 407.

[23] T.S. Van Kortryk, The Root of the Problem, https://hal.archives-ouvertes.fr/hal-02273293.

[24] C. Fox, The asymptotic expansion of integral functions defined by generalized hypergeometric series, Proc. Lond. Math. Soc. 27 (1928) 389.

[25] P.G.O. Freund and Y. Nambu, Scalar field coupled to the trace of the energy-momentum tensor, Phys. Rev. 174 (1968) 1741 [InSPIRE].

[26] S. Deser and L. Halpern, Self-Coupled Scalar Gravitation, Gen. Rel. Grav. 1 (1970) 131 [INSPIRE]. 